\title{
Development of Real-Time Probe Current Calibration for Performing Quantitative STEM with a Cold Field-Emission Gun.
}

\author{
Stephen D. House ${ }^{1}$, C.T. Schamp ${ }^{2}$, Russell Henry ${ }^{2}$, Dong $\mathrm{Su}^{3}$, Eric Stach $^{3}$, and Judith C. Yang ${ }^{1}$ \\ 1. Dept. of Chemical and Petroleum Engineering, University of Pittsburgh, Pittsburgh, PA (USA) \\ 2. Hitachi High Technologies America, Inc., Dallas, TX (USA) \\ 3. Center for Functional Nanomaterials, Brookhaven National Laboratory, Upton, NY (USA)
}

The key to the rational design of catalysts is understanding the structure/reactivity relationship and the critical structural factors that impact catalytic reactions. In particular, the catalytic activity and effectiveness of nanoparticles are strongly dependent on the atomic-scale details of the 3D structure. Aberration-corrected transmission electron microscopy (TEM) is uniquely situated in its ability to provide direct structural, chemical, and compositional characterization at the atomic level. Structural ambiguity introduced by the 2D nature of TEM micrographs, however, can hinder the determination of the true 3D structure. Quantitative STEM offers an approach for extracting this information from a single high-angle annular dark-field scanning TEM (HAADF-STEM) micrograph. First demonstrated nearly two decades ago by Singhal et al. [1], the recent advancements in aberration-correction for STEM spurred a resurgence in interest in quantitative STEM when it was shown the technique could be performed with atomic resolution by LeBeau et al. [2]. Through careful calibration of the microscope and image, the contrast (scattered intensity) in the micrographs can be explicitly related back to the number of atoms involved in the scattering through comparison with image simulation, yielding information about the 3D structure [3] and composition [4] of the specimens.

The current quantitative STEM work has been performed using microscopes equipped with Schottky field-emission gun (FEG) electron sources. The highest-performance analytical STEMs, however, are equipped with cold FEG (CFEG) sources, since CFEGs offer superior spatial and temporal coherences, yielding higher spatial and energy resolutions for imaging and spectroscopy. This performance gain comes at the cost of reduced stability of the emission current, which, in a CFEG, decays in a continuous, non-linear fashion even image-to-image. This poses a challenge for quantitative STEM, as one of the key calibrations requires knowledge of the incident beam current to normalize image intensities into units of fractional beam current for comparison with image simulations [2,5]. The conventional approach to this calibration involves measuring the current at the beginning of a session and assuming it remains constant for subsequent imaging. This is unsuitable for a CFEG, as it can introduce significant error into the intensity calibration and resulting computation of $3 \mathrm{D}$ structure.

In this presentation we will discuss our method to overcome this issue by adapting the condenser aperture of a Hitachi HD-2700C STEM to act as a beam monitor to measure the incident probe current in real-time concurrent with STEM image acquisition. Equipment has been installed to enable the signal produced by electrons impinging on the aperture to be read in by image acquisition software. This beam monitor signal was calibrated against measurements of the probe current via a Faraday cup, Figure 1. Whenever a STEM image is subsequently acquired, a corresponding beam monitor signal image is simultaneously acquired. This allows each pixel in the STEM image to be calibrated for quantitative STEM analysis individually, using the instantaneous probe current incident on the specimen during that pixel's acquisition time. This method enables a more accurate calibration of intensity to be achieved on 
microscopes with CFEGs. The benefits of real-time beam current measurement also extend to other S/TEM techniques, such as Electron Energy-Loss Spectroscopy (EELS). [6]

References:

[1] A Singhal, et al., Ultramicroscopy 67 (1997), p. 191-206

[2] JM LeBeau, et al., Physical Review Letters 100 (2008), p. 20601

[3] L Jones, et al., Nano Letters 14 (2014), p. 6336-6341

[4] A Rosenauer, et al., Ultramicroscopy 109 (2009), p. 1171-1182

[5] H E, et al., Ultramicroscopy 133 (2013), p. 109-119

[6] This work was supported by DOE BES through grant DE FG02-03ER15476, and performed using the facilities at the Center for Functional Nanomaterials at Brookhaven National Laboratory, which is supported by DOE BES through contract DE-SC0012704.

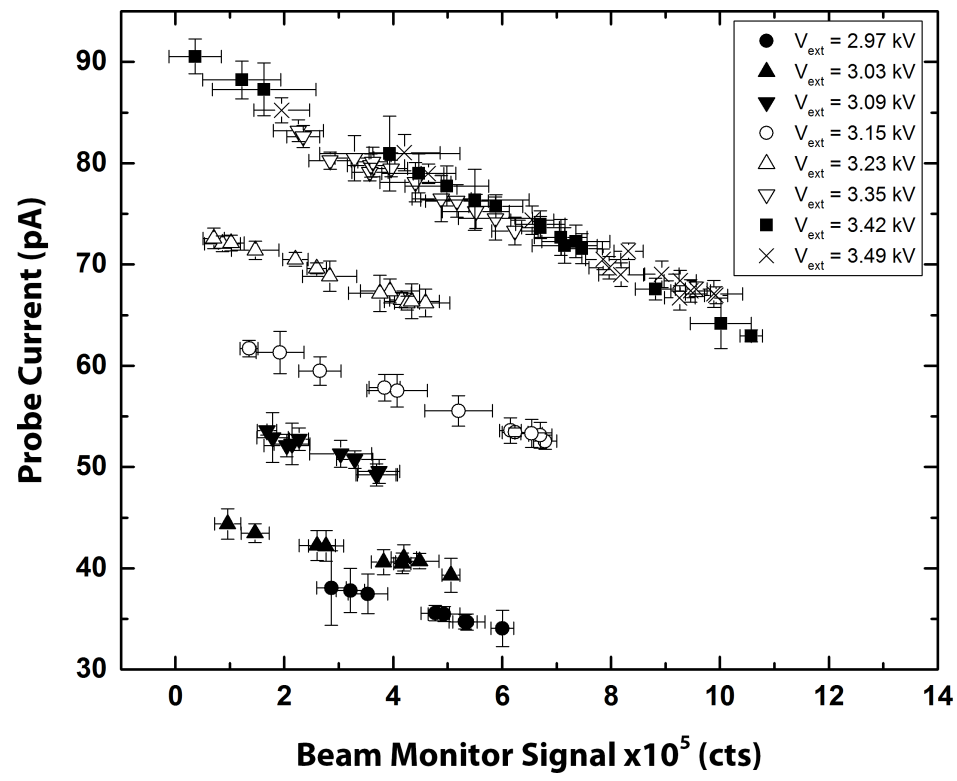

Figure 1. Comparison of probe current vs. beam monitor signal, showing the linear relationship between the two. The different symbols correspond to the extraction voltage. The standard deviations are plotted as error bars. 